\title{
BARGAINING OVER PUBLIC GOODS
}

\author{
Julio Dávila ${ }^{1}$ \\ Université catholique de Louvain, CORE, B-1348 Louvain-la-Neuve, Belgium \\ and \\ Paris School of Economics \\ Centre d'Economie de la Sorbonne, 106-112 Boulevard de l'Hopital , 75013 Paris, France
}

Jan Eeckhout

Department of Economics, University of Pennsylvania

160 McNeil Building, 3718 Locust Walk Philadelphia, Pennsylvania 19104-6297, USA

and

Department of Economics, Universitat Pompeu Fabra

Ramon Trias Fargas, 25-27, 08005 Barcelona, Spain

and

César Martinelli

Centro de Investigación Económica

Instituto Tecnológico Autónomo de México, Camino Santa Teresa 930, 10700 México DF, México

Abstract. In a simple public good economy, we propose a natural bargaining procedure, the equilibria of which converge to Lindahl allocations as the cost of bargaining vanishes. The procedure splits the decision over the allocation in a decision about personalized prices and a decision about output levels for the public good. Since this procedure does not assume price-taking behavior, it provides a strategic foundation for the personalized taxes inherent in the Lindahl solution to the public goods problem.

1 Julio Dávila gratefully acknowledges research funding as "Promoteur d'un M.I.S.-Mobilité Ulysse" from the Belgian F.R.S-FNRS) 


\section{INTRODUCTION}

The private provision of public goods in general leads to inefficient allocations in a competitive market environment. This inefficiency is often attributed to a missing market. If personalized markets could be created that individually price the public good for each agent, then a competitive equilibrium could implement an efficient allocation. For an economy with public goods, this outcome is known as a Lindahl equilibrium. Typically, however, a Lindahl equilibrium is deemed unrealistic because of a serious shortcoming: in the personalized markets upon which it rests the agents are assumed to be price-takers. Unfortunately, by the personalized nature of those markets, there is only one single agent on the demand side in each of them, which makes price-taking behavior of this single agent an utterly unrealistic assumption. In contrast, in this paper we propose a bargaining procedure that leads to an outcome arbitrarily close to a Lindahl allocation as the cost of bargaining vanishes, without the need of assuming price-taking behavior. As a matter of fact, the agents in our model have quite a lot of market power.

In the case of a missing market, as is the case in the presence of a public good, one way to allocate the surplus left unappropriated is through Coasian bargaining. As pointed out by Coase, as long as there remain gains from trade the parties involved have incentives to get together and strike a deal. The main feature of such bargaining is that it is decentralized (no benevolent government must intervene), and the extent to which the surplus can be allocated to the parties depends on the details of the bargaining protocol and on whether the bargaining is costly or not.

The study of this type of bargaining in legislatures has already been addressed in Baron and Ferejohn (1989), where a model is presented in which one of the different possible ways to divide a given pie is chosen by a vote according to the majority rule. It turns out that typically there exist many equilibria for this procedure. Banks and Duggan (2000) present a far more general model in which the space of alternatives is a compact, convex subset of a multidimensional Euclidean space. They consider arbitrary voting rules and prove the existence of stationary equilibria, the upper hemicontinuity of equilibrium proposals in structural and preference parameters, and a core equivalence result. While several of these bargaining set-ups encompass economies with public goods, they differ from our own set-up in that ours implicitly imposes constraints on what the proposer is able to offer to other agents. We believe these constraints reflect in a natural way the sharing of power in a bargaining

Typeset by $\mathcal{A} \mathcal{M} \mathcal{S}-\mathrm{T}_{\mathrm{E}} \mathrm{X}$ 
situation over both the output level and the financing of a public good. As a consequence of imposing such constraints on the offers, the bargaining protocol considered here attains in the limit the outcome that would result from completing the missing markets. This holds without resorting to the heroic assumption of price-taking behavior with respect to personalized prices.

Specifically, we model the collective decision making process with respect to the provision of public goods as a sequential bargaining game. The sequential nature of the game reflects a realistic feature of the power of setting the agenda of the negotiation. The proposal and acceptance decisions are endogenous. Agents can reject a proposal and have it modified in their turn. Because of the cost of any delay in reaching an agreement, the bargaining outcome will in general be inefficient, thus reflecting the power of setting the agenda: when there is impatience for reaching an agreement, the agent who makes an offer that is accepted will extract more rents at the cost of inefficiency. As the impatience or cost of a delay vanishes, those rents disappear and the allocation is efficient and independent of who had the power of setting the agenda.

We show that, despite the fact that output decisions for public goods and their mode of financing are often the result of a political process rife with opportunities for strategic behavior, the Lindahl allocations can be implemented without assuming price-taking behavior with respect to personalized prices. This is obtained precisely through strategic bargaining by the parties over the financing of the public good. ${ }^{1}$ In order to show this we take here a first step towards modelling the political process behind output and financing decisions with regard to public goods as a sequential bargaining game of complete information.

We consider an economy with any finite number of public goods and private goods (not necessarily the same number of each). There are two agents in this economy who take turns alternatingly (as in Rubinstein (1982)) to propose a maximum level of provision of each of the public goods and a way to split between them the cost of financing any level of the public goods to be provided up to the proposed maxima (this amounts to proposing personalized prices or taxes). The other agent can then

\footnotetext{
${ }^{1}$ The mixed competitive mechanisms proposed in Groves and Ledyard (1977) obviously can efficiently allocate private and public goods to coincide with the Lindahl allocation. However, those direct mechanisms both rely on a centralized mechanism designer and they may involve complicated mechanisms. The virtue here is that an efficient allocation of private and public goods is obtained through a decentralized bargaining procedure. It is simple: it relies explicitly on personalized contributions (taxes) and incorporates the notion that agenda setters have power to extract more rents when bargaining is costly.
} 
either accept or reject the proposal. In case of acceptance, this other agent chooses the amount of each public good to be provided (subject to the maximum amount offered in the proposal). Each agent pays for the public goods according to the personalized prices agreed upon. The levels of public goods and their financing are fixed thereafter so the game is effectively over. If instead the other agent rejects the proposal, then it is his turn to make a proposal himself of a new maximum amounts and personalized prices, and so on.

Within this set-up we show that, as the discount factors of each agent in the economy converge to one, the allocation of any stationary subgame perfect equilibrium converges to a Lindahl equilibrium allocation. In particular, we show first that, for infinitely patient agents (i.e., for discount factors equal to 1), the set of Lindahl allocations coincides with the set of stationary subgame perfect equilibrium allocations of the alternating-offer bargaining game described above. Then we establish the upper hemicontinuity of the correspondence mapping, to each pair of discount factors, the set of stationary subgame perfect equilibrium allocations of the bargaining game, and in particular its upper hemicontinuity for discount factors $\delta^{A}=\delta^{B}=1$. The conclusion then follows from these two results.

In this simple set-up, unanimity plays an important role in our modeling. In effect, a tax proposal can be adopted only if it is acceptable to every agent. This has the virtue of conferring a Wicksellian character to the Lindahl solution to the public goods problem. In a classical contribution to the theory of public finance, Wicksell (1896) proposed unanimity as the criterion for just taxation. The game we propose incorporates the need for achieving consensus to effect tax proposals that to some extent may characterize budgetary procedures in political regimes with multiple checks and balances or in parliamentary democracies without a majority party. Inefficiencies associated with bargaining are shown to disappear as agents become increasingly patient. Thus, a Wicksellian procedure turns out to be consistent with a Lindahl result. Of course, this result depends on the assumption of complete information.

The results of this paper parallel results obtained in Dávila and Eeckhout (2008) for economies without public goods. In that paper the authors provide a bargaining foundation for Walrasian equilibria in a two-agent exchange economy in which agents are not price-takers. The strategy of the proofs here is similar, although the presence of public goods in co-existence with private goods introduces significant technical differences for the proofs provided in Dávila and Eeckhout (2008) not to go through straightforwardly. In particular, the public goods problem augments the 
private goods problem in two ways. First, in the private goods economy, all prices are common across agents and consumptions for each good are agent specific. In the public goods economy we add to the previous setup prices that are agent specific for each public good, while the consumption of each public good is common across agents. Second, the presence of public goods naturally introduces production in the economy. In this sense the results provided here do not follow as a corollary from those in Dávila and Eeckhout (2008).

More importantly, we think that looking for a bargaining foundation for the Lindahl equilibrium is an even more natural question to address given that the decision about the provision of public goods typically takes place within legislatures. From this perspective, we think that the proposed setup captures well the problem faced by two parties with known preferences that try to reach an agreement on the provision of some public goods. Moreover, this bargaining foundation addresses one of the main critiques of the Lindahl equilibrium notion, namely the inevitable market power that the asumption of personalized markets for the public goods entails. We discuss possible extensions of our results in the final remarks.

\section{THE MODEL}

We consider a public good economy consisting of two agents $A$ and $B$, and an arbitrary number $n+m$ of goods: $n$ private goods $x_{1}, \ldots, x_{n}$ and $m$ public goods $y_{1}, \ldots, y_{m}$. The agents are infinitely lived and time is discrete. For each agent $i=A, B$, let $x^{i} \in \mathbb{R}_{+}^{n}$ be $i$ 's consumption of private goods, and let $y \in \mathbb{R}_{+}^{m}$ be their common consumption of public goods. The agents have preferences over the two types of goods represented by standard utility functions $u^{A}\left(x^{A}, y\right)$ and $u^{B}\left(x^{B}, y\right)$, that is to say non-negative, $C^{2}$, monotone, differentiably strictly quasi-concave, ${ }^{2}$ and well-behaved at the boundary of $\mathbb{R}_{+}^{n+m} .3$ This good behavior at the boundary guarantees the possibility of generating a surplus by the production of public goods, i.e. at a Lindahl equilibrium necessarily $y>0$. The agents are endowed with amounts $e^{i}$ (with total endowment $e=e^{A}+e^{B}$ ) of the private goods. As a normalization, we assume the initial amount of the public goods is zero. A linear technology $M \in \mathbb{R}^{n \times m}$ allows to produce each public good $y_{j}$ by means of the private goods, requiring $m_{i j}$ units of private good $x_{i}$ for each unit of $y_{j}$, for all

${ }^{2}$ That is to say, for all $i=A, B$, for all $(x, y) \in \mathbb{R}_{++}^{n+m}, D^{2} u^{i}(x, y)$ is negative definite in the space orthogonal to $D u^{i}(x, y)$.

${ }^{3}$ That is to say, for all $i=A, B$, and all $x_{j}, y_{k}$, it holds true that $\lim _{x_{h} \rightarrow 0} D_{x_{h}} u^{i}(x, y)=\infty$, and $\lim _{y_{k} \rightarrow 0} D_{y_{k}} u^{i}(x, y)=\infty$. 
$i=1, \ldots, n$.

We consider an alternating-offers bargaining game. In any given period prior to an agreement, an agent $i$ makes an offer consisting of a vector $p_{i}=\left(p_{x i}, p_{y i}\right)$ of prices for the other agent (in terms of, say, the private good $x_{1}$ ) and an upper bound $q_{i}$ on the other agent's contribution of private goods to the provision of public goods or, equivalently, to the provision of public goods itself. After receiving an offer the other agent can either accept it or reject it. In case of acceptance, the accepting agent chooses his consumption of private goods and the quantities of public goods to be provided subject to the accepted prices $p_{i}$ and upper bound on trades $q_{i}$.

This procedure is repeated until a proposal is accepted. The utility of each agent $i$ is discounted in each iteration by a positive discount factor $\delta^{i}$ not bigger than 1 . The utility of never reaching an agreement is 0 .

\section{The stationary subgame Perfect (SSP) Equilibrium ALLOCATIONS}

We consider first the stationary subgame perfect equilibria without delay of the bargaining game, that is the SSP equilibria in which no agent has incentives to reject the offer received. Indeed, as argued in Section 6 below, there does not exist any SSP equilibrium with delay if the agents are impatient, that is to say if $\delta^{A}, \delta^{B}<1$.

The SSP equilibria with no delay are characterized by a pair of offers $\left(p_{A}, q_{A}\right)$ and $\left(p_{B}, q_{B}\right)$, consisting each of a vector of prices and a maximum amount for the contributions of private goods, such that $\left(p_{A}, q_{A}\right)$ maximizes the utility that $A$ obtains from $B$ 's immediate acceptance, subject to the constraint that it is indeed in $B$ 's interest to accept $A$ 's offer, and similarly for $\left(p_{B}, q_{B}\right)$.

Formally, $\left(p_{A}, q_{A}\right)$ solves

$$
\max u^{A}\left(e^{A}-\tilde{x}^{B}\left(p_{A}, q_{A}\right)-M \tilde{y}^{B}\left(p_{A}, q_{A}\right), \tilde{y}^{B}\left(p_{A}, q_{A}\right)\right)
$$

subject to the constraint

$$
\begin{aligned}
u^{B}\left(\tilde{x}^{B}\left(p_{A}, q_{A}\right), \tilde{y}^{B}\left(p_{A}, q_{A}\right)\right) & \geq \\
& \delta^{B} u^{B}\left(e^{B}-\tilde{x}^{A}\left(p_{B}, q_{B}\right)-M \tilde{y}^{A}\left(p_{B}, q_{B}\right), \tilde{y}^{A}\left(p_{B}, q_{B}\right)\right),
\end{aligned}
$$


where $\tilde{x}^{B}\left(p_{A}, q_{A}\right), \tilde{y}^{B}\left(p_{A}, q_{A}\right)$ is the solution to

$$
\begin{gathered}
\max u^{B}(x, y) \\
p_{A} \cdot\left(x-e^{B}, y\right) \leq 0 \\
\left\|\left(x-e^{B}, y\right)\right\| \leq q_{A},
\end{gathered}
$$

where $\|\cdot\|$ stands for the Euclidean norm, ${ }^{4}$ and symmetrically for $\left(p_{B}, q_{B}\right)$.

Note that a SSP equilibrium without delay can equivalently be characterized by the allocations effectively offered by the agents. In effect, we establish in Lemma 1 in the Appendix that, conditional on immediate acceptance, an offer by $A$ of $\left(p_{A}, q_{A}\right)$ amounts to offering $B$ the bundle $\left(\tilde{x}^{B}\left(p_{A}, q_{A}\right), \tilde{y}^{B}\left(p_{A}, q_{A}\right)\right)$, which is characterized by satisfying the condition

$$
\left(u_{x}^{B}\left(x^{B}, y\right), u_{y}^{B}\left(x^{B}, y\right)\right)\left(\begin{array}{c}
x^{B}-e^{B} \\
y
\end{array}\right) \geq 0 .
$$

Conversely, any bundle $\left(x^{B}, y\right)$ satisfying the previous inequality is a solution to $B$ 's problem above for some offer $\left(p_{A}, y_{A}\right)$ by $A$.

As a consequence, a SSP equilibrium without delay can also be characterized by allocations $\left(x_{A}^{A}, x_{A}^{B}, y_{A}\right)$ and $\left(x_{B}^{A}, x_{B}^{B}, y_{B}\right)$, proposed by $A$ and $B$ respectively, such that $\left(x_{A}^{A}, x_{A}^{B}, y_{A}\right)$ solves

$$
\begin{array}{r}
\max u^{A}\left(x^{A}, y\right) \\
\left(u_{x}^{B}\left(x^{B}, y\right), u_{y}^{B}\left(x^{B}, y\right)\right)\left(\begin{array}{c}
x^{B}-e^{B} \\
y
\end{array}\right) \geq 0 \\
u^{B}\left(x^{B}, y\right) \geq \delta^{B} u^{B}\left(x_{B}^{B}, y_{B}\right) \\
x^{A}+x^{B}+M y=e^{A}+e^{B}
\end{array}
$$

given $\left(x_{B}^{A}, x_{B}^{B}, y_{B}\right)$, and $\left(x_{B}^{A}, x_{B}^{B}, y_{B}\right)$ solves

$$
\begin{array}{r}
\max u^{B}\left(x^{B}, y\right) \\
\left(u_{x}^{A}\left(x^{A}, y\right), u_{y}^{A}\left(x^{A}, y\right)\right)\left(\begin{array}{c}
x^{A}-e^{A} \\
y
\end{array}\right) \geq 0 \\
u^{A}\left(x^{A}, y\right) \geq \delta^{A} u^{A}\left(x_{A}^{A}, y_{A}\right) \\
x^{A}+x^{B}+M y=e^{A}+e^{B}
\end{array}
$$

\footnotetext{
${ }^{4}$ This choice is done for analytical convenience. As a matter of fact, being in a finite-dimension vector space, all norms are equivalent, so that the choice of the norm in equation (4) is inessential.
} 
given $\left(x_{A}^{A}, x_{A}^{B}, y_{A}\right)$.

At this point, it may be worth to clarify the main difference between Rubinstein's setup and ours. Note that in choosing their offers the agents are constrained not only to guarantee each other the continuation value at each stage, but also that this value is computed only for prices and quotas that are subgame perfect. A consequence of introducing this additional constraint is that, when the agents are impatient, there may remain surplus still to be distributed at a SSP equilibrium. In Rubinstein's setup this is ruled out since there, all the feasible utility profiles are available for negotiation. Here the utility profiles available are constrained to be those attainable as SSP equilibrium outcomes of bargaining over prices and maximum provisions of public goods. In fact, without constraint (5) in problem (6) and the analogous constraint in problem (7), each proposal would be Pareto efficient like in Rubinstein. In Section 6 below we show that such inefficiency vanishes as the agents become arbitrarily patient.

\section{The Lindahl allocations}

A Lindahl equilibrium consists of a vector of private good prices $p_{x}$, a vector of personalized prices for the public goods $p_{y}^{A}, p_{y}^{B}$, and an allocation $\left(x^{A}, x^{B}, y\right)$ such that

(1) the allocation is feasible, i.e. $x^{A}, x^{B}, y \geq 0$ and

$$
x^{A}+x^{B}+M y=e^{A}+e^{B} .
$$

(2) each agent's consumption $\left(x^{i}, y\right), i=A, B$, maximizes his utility given $p_{x}$ and $p_{y}^{i}$, i.e.

$$
\begin{aligned}
\left(u_{x}^{i}\left(x^{i}, y\right), u_{y}^{i}\left(x^{i}, y\right)\right) & =\lambda^{i}\left(p_{x}, p_{y}^{i}\right) \\
p_{x}\left(x^{i}-e^{i}\right)+p_{y}^{i} y & =0
\end{aligned}
$$

for some $\lambda^{i}>0$.

(3) the production of public goods maximizes profits, which implies, when $y \gg$ $0,{ }^{5}$

$$
p_{y}^{A}+p_{y}^{B}-p_{x} M=0
$$

\footnotetext{
${ }^{5}$ The production of an amount 0 for some public good cannot be an equilibrium outcome given that the utility functions behavior at the boundary guarantees interior solutions for positive prices.
} 
Therefore, a Lindahl equilibrium allocation $\left(x^{A}, x^{B}, y\right)$ is a feasible allocation such that $u_{x}^{A}\left(x^{A}, y\right)$ and $u_{x}^{B}\left(x^{B}, y\right)$ are colinear, and it allocates to each agent $i=A, B$ his demand $\left(x^{i}, y\right)$ at his personalized relative prices, implicitly equal to the marginal rates of substitution determined by his marginal utilities at $\left(x^{i}, y\right)$, i.e.

$$
\left(u_{x}^{i}\left(x^{i}, y\right), u_{y}^{i}\left(x^{i}, y\right)\right)\left(\begin{array}{c}
x^{i}-e^{i} \\
y
\end{array}\right)=0 .
$$

For the case of one private good and one public good produced with a 1-unit linear technology, Lindahl equilibrium allocations can be represented in a Kolm triangle, the public goods equivalent of the Edgeworth box of a private goods exchange economy (see Figure 1 below; Thomson (1999) provides a useful presentation and discussion of the Kolm triangle). The Kolm triangle assumes a linear production technology requiring one unit of the private good for each unit of the public good. The height of the triangle represents the initial total endowment of the private good. The orthogonal distance from any point within the triangle to each of its sides represents each agent's allocation of the private good $\left(x^{A}\right.$ and $\left.x^{B}\right)$, while the vertical distance to the base of the triangle represents their common consumption $y$ of the public good. Thus, at the initial endowment $\left(e^{A}, e^{B}\right)$ there is no provision of the public good.

Figure 1

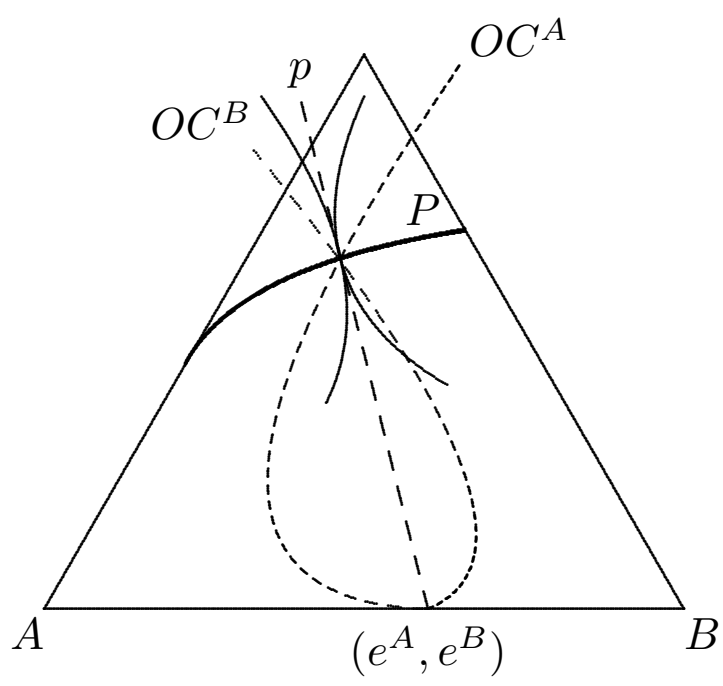

The personalized prices $p^{A}$ and $p^{B}$ represent the terms of exchange of one unit of public good for $p^{A}$ units of the private good from agent $A$ and $p^{B}$ units of private 
good from agent $B$. A balanced budget where the total contributions equal the total cost of production of the public good implies that for all $y, \sum_{i=1}^{I} p^{i} y=y$. For a given price schedule $p$, the offer curve $O C^{i}$ gives the optimal amount of public good and private good demanded by agent $i$ at those terms of trade. An intersection of the offer curves represents then optimal quantities of the public good and the private good consumed by the agents given a vector of personalized prices that balances the budget. This corresponds to a Lindahl equilibrium allocation. There are of course other efficient allocation represented by the Pareto set $P$, but they are not attainable by means of price schedules starting from the initial endowment $\left(e^{A}, e^{B}\right)$.

\section{SSP EQUILIBRIUM ALLOCATIONS ARE \\ LiNDAHL ALLOCATIONS WHEN $\delta^{A}=\delta^{B}=1$}

First we show that for infinitely patient agents, that is, when the discount factors $\delta^{A}$ and $\delta^{B}$ are 1 , the Lindahl equilibrium allocations, and only these allocations, are offered at a stationary subgame perfect equilibrium of the bargaining game above. For discount factors equal to one the only SSP equilibrium allocation is the Lindahl allocation. This is quite remarkable and in sharp contrast with the standard Rubinstein bargaining equilibrium, where the entire Pareto frontier is an equilibrium when there is no discounting.

Theorem 1. When $\delta^{A}=\delta^{B}=1$, at every SSP equilibrium with immediate acceptance the agents' offers lead to the same allocation. Moreover, this allocation is a Lindahl equilibrium allocation. Conversely, every Lindahl equilibrium allocation is the allocation offered by the two agents at some SSP equilibrium without delay.

Proof. Let $\left(x_{A}^{A}, x_{A}^{B}, y_{A}\right)$ and $\left(x_{B}^{A}, x_{B}^{B}, y_{B}\right)$ be the feasible allocations resulting from $B$ 's (resp. $A$ 's) acceptance of $A$ 's (resp. $B$ 's) offer of price and maximum provisions of public goods at a SSP equilibrium with immediate acceptance for infinitely patient players. That is, let $\left(x_{A}^{A}, x_{A}^{B}, y_{A}\right)$ and $\left(x_{B}^{A}, x_{B}^{B}, y_{B}\right)$ be such that 


$$
\begin{array}{r}
\left(x_{A}^{A}, x_{A}^{B}, y_{A}\right) \in \arg \max _{x^{A}, x^{B}, y} u^{A}\left(x^{A}, y\right) \\
\left(u_{x}^{B}\left(x^{B}, y\right), u_{y}^{B}\left(x^{B}, y\right)\right)\left(\begin{array}{c}
x^{B}-e^{B} \\
y
\end{array}\right) \geq 0 \\
u^{B}\left(x^{B}, y\right) \geq u^{B}\left(x_{B}^{B}, y_{B}\right) \\
x^{A}+x^{B}+M y=e^{A}+e^{B} \\
\text { given }\left(x_{B}^{A}, x_{B}^{B}, y_{B}\right),
\end{array}
$$

and

$$
\begin{array}{r}
\left(x_{B}^{A}, x_{B}^{B}, y_{B}\right) \in \arg \max _{x^{A}, x^{B}, y} u^{B}\left(x^{B}, y\right) \\
\left(u_{x}^{A}\left(x^{A}, y\right), u_{y}^{A}\left(x^{A}, y\right)\right)\left(\begin{array}{c}
x^{A}-e^{A} \\
y
\end{array}\right) \geq 0 \\
u^{A}\left(x^{A}, y\right) \geq u^{A}\left(x^{A}, y\right) \\
x^{A}+x^{B}+M y=e^{A}+e^{B} \\
\text { given }\left(x_{A}^{A}, x_{A}^{B}, y_{A}\right) .
\end{array}
$$

Then, from the from the first-order conditions, there exist multipliers $\lambda^{A}, \mu^{A}, \lambda^{B}$, $\mu^{B} \geq 0$ and $\nu^{A}, \nu^{B}$ such that

$$
\begin{array}{r}
\left(\begin{array}{c}
u_{x}^{A}\left(x_{A}^{A}, y_{A}\right) \\
0 \\
u_{y}^{A}\left(x_{A}^{A}, y_{A}\right)
\end{array}\right)+\lambda^{A}\left(\begin{array}{c}
0 \\
u_{x}^{B}\left(x_{A}^{B}, y_{A}\right) \\
u_{y}^{B}\left(x_{A}^{B}, y_{A}\right)
\end{array}\right) \\
+\mu^{A}\left(\begin{array}{c}
u_{x}^{B}\left(x_{A}^{B}, y_{A}\right)+u_{x x}^{B}\left(x_{A}^{B}, y_{A}\right)\left(x_{A}^{B}-e^{B}\right)+u_{y x}^{B}\left(x_{A}^{B}, y_{A}\right) y_{A} \\
u_{y}^{B}\left(x_{A}^{B}, y_{A}\right)+u_{x y}^{B}\left(x_{A}^{B}, y_{A}\right)\left(x_{A}^{B}-e^{B}\right)+u_{y y}^{B}\left(x_{A}^{B}, y_{A}\right) y_{A}
\end{array}\right) \\
+\sum_{i=1}^{n} \nu_{i}^{A}\left(\begin{array}{c}
e_{i} \\
e_{i} \\
m_{i}^{t}
\end{array}\right)=0
\end{array}
$$

where $m_{i}^{t}$. is the $i$-th row of $M$ transposed as a column. Equivalently, eliminating the multipliers $\nu_{i}^{A}$,

$$
\begin{gathered}
\left(\begin{array}{c}
u_{x}^{A}\left(x_{A}^{A}, y_{A}\right) \\
M^{t} u_{x}^{A}\left(x_{A}^{A}, y_{A}\right)-u_{y}^{A}\left(x_{A}^{A}, y_{A}\right)
\end{array}\right)=\lambda^{A}\left(\begin{array}{c}
u_{x}^{B}\left(x_{A}^{B}, y_{A}\right) \\
u_{y}^{B}\left(x_{A}^{B}, y_{A}\right)
\end{array}\right) \\
+\mu^{A}\left(\begin{array}{c}
u_{x}^{B}\left(x_{A}^{B}, y_{A}\right)+u_{x x}^{B}\left(x_{A}^{B}, y_{A}\right)\left(x_{A}^{B}-e^{B}\right)+u_{y x}^{B}\left(x_{A}^{B}, y_{A}\right) y_{A} \\
u_{y}^{B}\left(x_{A}^{B}, y_{A}\right)+u_{x y}^{B}\left(x_{A}^{B}, y_{A}\right)\left(x_{A}^{B}-e^{B}\right)+u_{y y}^{B}\left(x_{A}^{B}, y_{A}\right) y_{A}
\end{array}\right) \\
10
\end{gathered}
$$


and similarly

$$
\begin{aligned}
& \left(\begin{array}{c}
u_{x}^{B}\left(x_{B}^{B}, y_{B}\right) \\
M^{t} u_{x}^{B}\left(x_{B}^{B}, y_{B}\right)-u_{y}^{B}\left(x_{B}^{B}, y_{B}\right)
\end{array}\right)=\lambda^{B}\left(\begin{array}{c}
u_{x}^{A}\left(x_{B}^{A}, y_{B}\right) \\
u_{y}^{A}\left(x_{B}^{A}, y_{B}\right)
\end{array}\right) \\
& +\mu^{B}\left(\begin{array}{c}
u_{x}^{A}\left(x_{B}^{A}, y_{B}\right)+u_{x x}^{A}\left(x_{B}^{A}, y_{B}\right)\left(x_{B}^{A}-e^{A}\right)+u_{y x}^{A}\left(x_{B}^{A}, y_{B}\right) y_{B} \\
u_{y}^{A}\left(x_{B}^{A}, y_{B}\right)+u_{x y}^{A}\left(x_{B}^{A}, y_{B}\right)\left(x_{B}^{A}-e^{A}\right)+u_{y y}^{A}\left(x_{B}^{A}, y_{B}\right) y_{B}
\end{array}\right)
\end{aligned}
$$

Assume that $\left(x_{A}^{A}, x_{A}^{B}, y_{A}\right) \neq\left(x_{B}^{A}, x_{B}^{B}, y_{B}\right)$. Since at a SSP equilibrium with $\delta^{A}=$ $\delta^{B}=1$ the constraints

$$
\begin{aligned}
& u^{A}\left(x_{B}^{A}, y_{B}\right) \geq u^{A}\left(x_{A}^{A}, y_{A}\right) \\
& u^{B}\left(x_{A}^{B}, y_{A}\right) \geq u^{B}\left(x_{B}^{B}, y_{B}\right)
\end{aligned}
$$

are binding, ${ }^{6}$ and hence both allocations are on the same indifference surface for both agents, ${ }^{7}$ then none of these two allocations can be efficient while being different. Therefore the efficiency condition that

$$
\left(\begin{array}{c}
u_{x}^{A}\left(x^{A}, y\right) \\
u_{y}^{A}\left(x^{A}, y\right)
\end{array}\right) \text { and }\left(\begin{array}{c}
u_{x}^{B}\left(x^{B}, y\right) \\
M^{t} u_{x}^{B}\left(x^{B}, y\right)-u_{y}^{B}\left(x^{B}, y\right)
\end{array}\right)
$$

are collinear cannot hold neither at $\left(x_{A}^{A}, x_{A}^{B}, y_{A}\right)$ nor at $\left(x_{B}^{A}, x_{B}^{B}, y_{B}\right)$. In particular, there exist $h, k$ such that

$$
\frac{u_{y_{k}}^{A}\left(x_{B}^{A}, y_{B}\right)}{u_{x_{h}}^{A}\left(x_{B}^{A}, y_{B}\right)} \neq \frac{\sum_{i=1}^{n} m_{i k} u_{x_{i}}^{B}\left(x_{B}^{B}, y_{B}\right)-u_{y_{k}}^{B}\left(x_{B}^{B}, y_{B}\right)}{u_{x_{h}}^{B}\left(x_{B}^{B}, y_{B}\right)}
$$

Suppose

$$
\frac{u_{y_{k}}^{A}\left(x_{B}^{A}, y_{B}\right)}{u_{x_{h}}^{A}\left(x_{B}^{A}, y_{B}\right)}<\frac{\sum_{i=1}^{n} m_{i k} u_{x_{i}}^{B}\left(x_{B}^{B}, y_{B}\right)-u_{y_{k}}^{B}\left(x_{B}^{B}, y_{B}\right)}{u_{x_{h}}^{B}\left(x_{B}^{B}, y_{B}\right)}
$$

We claim that if (16) holds, then equation (13) cannot be satisfied for non-negative multipliers. (A similar argument shows that if the inequality is reversed, then equation (12) cannot be satisfied for nonnegative multipliers.)

${ }^{6}$ For instance, since $\left(x_{A}^{A}, x_{A}^{B}, y_{A}\right)$ satisfies $D u^{A}\left(x^{A}, y\right)\left(x^{A}-e^{A}, y\right) \geq 0$ (agent $A$ will never choose at equilibrium to let $B$ ask for a provision of public goods bigger than the one necessary to attain $A$ 's demand at the implicit prices), then $u^{B}\left(x_{A}^{B}, y_{A}\right) \leq u^{B}\left(x_{B}^{B}, y_{B}\right)$ holds as well.

${ }^{7}$ For this step to hold true it is crucial that $\delta^{A}=\delta^{B}=1$. 
In effect, since $\left(\lambda^{B}, \mu^{B}\right)$ must solve (13) above, then it must also solve

$$
\begin{aligned}
& \left(\begin{array}{c}
u_{x_{h}}^{B}\left(x_{B}^{B}, y_{B}\right) \\
\sum_{i=1}^{n} m_{i k} u_{x_{i}}^{B}\left(x_{B}^{B}, y_{B}\right)-u_{y_{k}}^{B}\left(x_{B}^{B}, y_{B}\right)
\end{array}\right)=\lambda^{B}\left(\begin{array}{c}
u_{x_{h}}^{A}\left(x_{B}^{A}, y_{B}\right) \\
u_{y_{k}}^{A}\left(x_{B}^{A}, y_{B}\right)
\end{array}\right) \\
& +\mu^{B}\left(\begin{array}{c}
u_{x_{h}}^{A}\left(x_{B}^{A}, y_{B}\right)+u_{x_{h} x_{h}}^{A}\left(x_{B}^{A}, y_{B}\right)\left(x_{B}^{A}-e^{A}\right)+u_{y_{k} x_{h}}^{A}\left(x_{B}^{A}, y_{B}\right) y_{B} \\
u_{y_{k}}^{A}\left(x_{B}^{A}, y_{B}\right)+u_{x_{h} y_{k}}^{A}\left(x_{B}^{A}, y_{B}\right)\left(x_{B}^{A}-e^{A}\right)+u_{y_{k} y_{k}}^{A}\left(x_{B}^{A}, y_{B}\right) y_{B}
\end{array}\right)
\end{aligned}
$$

In particular, $\mu^{B}$ is equal to

$$
\frac{\left|\begin{array}{cc}
u_{x_{h}}^{A}\left(x_{B}^{A}, y_{B}\right) & u_{x_{h}}^{B}\left(x_{B}^{B}, y_{B}\right) \\
u_{y_{k}}^{A}\left(x_{B}^{A}, y_{B}\right) & \sum_{i=1}^{n} m_{i k} u_{x_{i}}^{B}\left(x_{B}^{B}, y_{B}\right)-u_{y_{k}}^{B}\left(x_{B}^{B}, y_{B}\right)
\end{array}\right|}{\left|\begin{array}{lll}
u_{x_{h}}^{A}\left(x_{B}^{A}, y_{B}\right) & u_{x_{h}}^{A}\left(x_{B}^{A}, y_{B}\right)+u_{x_{h} x_{h}}^{A}\left(x_{B}^{A}, y_{B}\right)\left(x_{B}^{A}-e^{A}\right)+u_{y_{k} x_{h}}^{A}\left(x_{B}^{A}, y_{B}\right) y_{B} \\
u_{y_{k}}^{A}\left(x_{B}^{A}, y_{B}\right) & u_{y_{k}}^{A}\left(x_{B}^{A}, y_{B}\right)+u_{x_{h} y_{k}}^{A}\left(x_{B}^{A}, y_{B}\right)\left(x_{B}^{A}-e^{A}\right)+u_{y_{k} y_{k}}^{A}\left(x_{B}^{A}, y_{B}\right) y_{B}
\end{array}\right|}
$$

Since $\mu^{B} \geq 0$, and the numerator is strictly positive according to (16), so must be the denominator. But the denominator is equivalent to

$$
\begin{aligned}
& \left(-u_{y_{k}}^{A}\left(x_{B}^{A}, y_{B}\right) \quad u_{x_{h}}^{A}\left(x_{B}^{A}, y_{B}\right)\right) \\
& \cdot\left[\left(\begin{array}{c}
u_{x_{h}}^{A}\left(x_{B}^{A}, y_{B}\right) \\
u_{y_{k}}^{A}\left(x_{B}^{A}, y_{B}\right)
\end{array}\right)+\left(\begin{array}{cc}
u_{x_{h} x_{h}}^{A}\left(x_{B}^{A}, y_{B}\right) & u_{x_{h} y_{k}}^{A}\left(x_{B}^{A}, y_{B}\right) \\
u_{y_{k} x_{h}}^{A}\left(x_{B}^{A}, y_{B}\right) & u_{y_{k} y_{k}}^{A}\left(x_{B}^{A}, y_{B}\right)
\end{array}\right)\left(\begin{array}{c}
x_{h B}^{A}-e_{h}^{A} \\
y_{k B}
\end{array}\right)\right],
\end{aligned}
$$

which is negative since the first scalar product is null and the Hessian of $u^{A}$ at $\left(x_{B}^{A}, y_{B}\right)$ is semi-definite negative in the space orthogonal to the gradient of $u^{A}$ at $\left(x_{B}^{A}, y_{B}\right)$ and hence also to any $\left(0, \ldots, 0, x_{h B}^{A}-e_{h}^{A}, 0, \ldots, 0, y_{k B}, 0, \ldots, 0\right)$ orthogonal to $\left(u_{x}^{A}\left(x_{B}^{A}, y_{B}\right), u_{y}^{A}\left(x_{B}^{A}, y_{B}\right)\right)$, i.e. for all $\left(x_{h B}^{A}-e_{h}^{A}, y_{k B}\right)$ such that

$$
u_{x_{h}}^{A}\left(x_{B}^{A}, y_{B}\right)\left(x_{h B}^{A}-e_{h}^{A}\right)+u_{y_{k}}^{A}\left(x_{B}^{A}, y_{B}\right) y_{k B}^{A}=0
$$

(Note that in this case $\left(-u_{y_{k}}^{A}\left(x_{B}^{A}, y_{B}\right), u_{x_{h}}^{A}\left(x_{B}^{A}, y_{B}\right)\right)$ is collinear to $\left(x_{h B}^{A}-e_{h}^{A}, y_{k B}\right)$ up to a positive constant.) It follows that if $\delta^{A}=\delta^{B}=1$ then at a SSP equilibrium with immediate acceptance the two agents offer the same allocation.

Let $x^{A}, x^{B}, y$ be the common allocation offered at a SSP equilibrium when $\delta^{A}=$ $\delta^{B}=1$. This allocation is therefore such that $u_{x}^{A}\left(x^{A}, y\right)$ and $u_{x}^{B}\left(x^{B}, y\right)$ are collinear, it is interior, in particular $y \gg 0$, given the boundary behavior of the utility functions $u^{i}$, and

$$
x^{A}+x^{B}+M y=e^{A}+e^{B},
$$




$$
\begin{array}{r}
\left(x^{A}, y\right) \in \arg \max u^{B}\left(e-\hat{x}^{A}-M \hat{y}, \hat{y}\right) \\
\left(u_{x}^{A}\left(\hat{x}^{A}, \hat{y}\right), u_{y}^{A}\left(\hat{x}^{A}, \hat{y}\right)\right)\left(\begin{array}{c}
\hat{x}^{A}-e^{A} \\
\hat{y}
\end{array}\right) \geq 0 \\
u^{A}\left(\hat{x}^{A}, \hat{y}\right) \geq u^{A}\left(e-x^{B}-M y, y\right) \\
\text { given } x^{B}, y,
\end{array}
$$

and

$$
\begin{array}{r}
\left(x^{B}, y\right) \in \arg \max u^{A}\left(e-\hat{x}^{B}-M \hat{y}, \hat{y}\right) \\
\left(u_{x}^{B}\left(\hat{x}^{B}, \hat{y}\right), u_{y}^{B}\left(\hat{x}^{B}, \hat{y}\right)\right)\left(\begin{array}{c}
\hat{x}^{B}-e^{B} \\
\hat{y}
\end{array}\right) \geq 0 \\
u^{B}\left(\hat{x}^{B}, \hat{y}\right) \geq u^{B}\left(e-x^{A}-M y, y\right) \\
\text { given } x^{A}, y .
\end{array}
$$

In effect, since $\delta^{A}=\delta^{B}=1$, should $u_{x}^{A}\left(x^{A}, y\right)$ and $u_{x}^{B}\left(x^{B}, y\right)$ not be collinear, then there would remain enough gains from trade to be exploited for any of the two agents to deviate making a Pareto-improving offer that the other agent would accept, contradicting that it is a SSP equilibrium alocation. Suppose also that

$$
\left(u_{x}^{A}\left(x^{A}, y\right), u_{y}^{A}\left(x^{A}, y\right)\right)\left(\begin{array}{c}
x^{A}-e^{A} \\
y
\end{array}\right)>0
$$

Then, since both $u^{A}$ and $u^{B}$ are concave,

$$
\begin{array}{r}
\left(x^{A}, y\right) \in \arg \max u^{B}\left(e-\hat{x}^{A}-M \hat{y}, \hat{y}\right) \\
u^{A}\left(\hat{x}^{A}, \hat{y}\right) \geq u^{A}\left(e-x^{B}-M y, y\right) \\
\text { given } x^{B}, y .
\end{array}
$$

Therefore, there exists $\lambda>0$ such that

$$
\begin{aligned}
\left(\begin{array}{c}
u_{x}^{A}\left(x^{A}, y\right) \\
u_{y}^{A}\left(x^{A}, y\right)
\end{array}\right) & =\lambda\left(\begin{array}{c}
u_{x}^{B}\left(x^{B}, y\right) \\
M^{t} u_{x}^{B}\left(x^{B}, y\right)-u_{y}^{B}\left(x^{B}, y\right)
\end{array}\right) \\
& =\lambda\left(\begin{array}{cc}
I_{n} & 0 \\
M^{t} & -I_{m}
\end{array}\right)\left(\begin{array}{c}
u_{x}^{B}\left(x^{B}, y\right) \\
u_{y}^{B}\left(x^{B}, y\right)
\end{array}\right),
\end{aligned}
$$

or equivalently,

$$
\left(u_{x}^{A}\left(x^{A}, y\right), u_{y}^{A}\left(x^{A}, y\right)\right)=\lambda\left(u_{x}^{B}\left(x^{B}, y\right), u_{y}^{B}\left(x^{B}, y\right)\right)\left(\begin{array}{cc}
I_{n} & M \\
0 & -I_{m}
\end{array}\right) .
$$


But from equations (21), (24) and (26) we get

$$
\left(u_{x}^{B}\left(x^{B}, y\right), u_{y}^{B}\left(x^{B}, y\right)\right)\left(\begin{array}{c}
x^{B}-e^{B} \\
y
\end{array}\right)<0,
$$

which contradicts equation (4) above. Therefore

$$
\left(u_{x}^{A}\left(x^{A}, y\right), u_{y}^{A}\left(x^{A}, y\right)\right)\left(\begin{array}{c}
x^{A}-e^{A} \\
y
\end{array}\right)=0
$$

and similarly

$$
\left(u_{x}^{B}\left(x^{B}, y\right), u_{y}^{B}\left(x^{B}, y\right)\right)\left(\begin{array}{c}
x^{B}-e^{B} \\
y
\end{array}\right)=0 .
$$

That is, the allocation proposed by both agents at a SSP equilibrium is on both agents' offer curves and such that $u_{x}^{A}\left(x^{A}, y\right)$ and $u_{x}^{B}\left(x^{B}, y\right)$ are collinear and, so that it is a Lindahl equilibrium allocation. ${ }^{8}$

Conversely, let $x^{A}, x^{B}, y$ be the allocation of a Lindahl equilibrium, i.e. an allocation such that

$$
\begin{gathered}
x^{A}+x^{B}+M y=e^{A}+e^{B}, \\
\left(u_{x}^{A}\left(x^{A}, y\right), u_{y}^{A}\left(x^{A}, y\right)\right)\left(\begin{array}{c}
x^{A}-e^{A} \\
y
\end{array}\right)=0,
\end{gathered}
$$

and

$$
\left(u_{x}^{B}\left(x^{B}, y\right), u_{y}^{B}\left(x^{B}, y\right)\right)\left(\begin{array}{c}
x^{B}-e^{B} \\
y
\end{array}\right)=0 .
$$

Let

$$
\left(p_{A}, q_{A}\right)=\left(\left(u_{x}^{B}\left(x^{B}, y\right), u_{y}^{B}\left(x^{B}, y\right)\right), y\right)
$$

and

$$
\left(p_{B}, q_{B}\right)=\left(\left(u_{x}^{A}\left(x^{A}, y\right), u_{y}^{A}\left(x^{A}, y\right)\right), y\right),
$$

${ }^{8}$ The additional condition for it to be a Lindahl equilibrium allocation, namely that $p_{y}^{A}+p_{y}^{B}-$ $p_{x} M=0$, follows from the satisfaction of the budget constraints (with the prices $p_{x}=u_{x}^{A}\left(x^{A}, y\right)$, $p_{y}^{A}=u_{y}^{A}\left(x^{A}, y\right)$, and $p_{y}^{B}=\mu u_{y}^{B}\left(x^{B}, y\right), \mu>0$ being the scalar for which $u_{x}^{A}\left(x^{A}, y\right)=\mu u_{x}^{B}\left(x^{B}, y\right)$ given their colinearity) and the fact that $y \gg 0$. 
so that $x^{A}=\tilde{x}^{A}\left(p_{B}, q_{B}\right), x^{B}=\tilde{x}^{B}\left(p_{A}, q_{A}\right)$ and $\tilde{y}^{A}\left(p_{B}, q_{B}\right)=y=\tilde{y}^{B}\left(p_{A}, q_{A}\right)$. It can be easily checked that the Lindahl allocation $\left(x^{A}, x^{B}, y\right)$ is the outcome of the following SSP equilibrium profile of strategies:

(1) $A$ offers $\left(p_{A}, q_{A}\right)$ whenever he has the opportunity to do so, and accepts only offers $(p, q)$ such that

$$
u^{A}\left(\tilde{x}^{A}(p, q), \tilde{y}^{A}(p, q)\right) \geq \delta^{A} u^{A}\left(\tilde{x}^{A}\left(p_{B}, q_{B}\right), \tilde{y}^{A}\left(p_{B}, q_{B}\right)\right)
$$

(2) $B$ offers $\left(p_{B}, q_{B}\right)$ whenever he has the opportunity to do so, and accepts only offers $(p, q)$ such that

$$
u^{B}\left(\tilde{x}^{B}(p, q), \tilde{y}^{B}(p, q)\right) \geq \delta^{B} u^{B}\left(\tilde{x}^{B}\left(p_{A}, q_{A}\right), \tilde{y}^{B}\left(p_{A}, q_{A}\right)\right) .
$$

Q.E.D.

\section{Convergence of SSP EqUilibrium Allocations TO LiNDAHL ALLOCATIONS AS $\delta^{A}, \delta^{B} \rightarrow 1$}

In the relevant case in which bargaining entails some frictions, so that the factors by which the agents discount future utilities are strictly smaller than 1, any SSP equilibrium allocation is still arbitrarily close to a Lindahl allocation if agents are patient enough. This is a consequence of the fact that the correspondence of SSP equilibrium allocations is upper hemicontinuous with respect to the agents' discount factors, as Theorem 2 next establishes. In the proof it suffices to consider only SSP equilibria in which the first offer gets accepted immediately, since there does not exist any SSP equilibrium with delay whenever $\delta^{A}, \delta^{B}<1$. $^{9}$

Theorem 2. Every SSP equilibrium allocation converges to a Lindahl allocation as $\delta^{A}, \delta^{B} \rightarrow 1$.

\footnotetext{
${ }^{9}$ Consider a candidate SSP equilibrium $\left(p_{A}, q_{A}\right)$ and $\left(p_{B}, q_{B}\right)$ in which, for instance, $B$ rejects and $A$ accepts. Let $\left(x_{B}^{A}, x_{B}^{B}, y_{B}\right)$ be the feasible allocation resulting from $A$ 's acceptance of $B$ 's offer of price and maximum provisions of public goods. Suppose that $\left(x_{B}^{A}, x_{B}^{B}, y_{B}\right)$ is efficient. Then $A$ could deviate offering himself $B$ 's offer instead, since $A$ will accept it anyway later, saving the cost of delay in reaching an agreement. Since $\left(x_{B}^{A}, x_{B}^{B}, y_{B}\right)$ is efficient, there is a price vector such that if offered this price and a nonbinding quota, $B$ would choose this allocation. Suppose that $\left(x_{B}^{A}, x_{B}^{B}, y_{B}\right)$ is inefficient. Then there is room for $A$ deviating and making an offer that is efficient and Pareto improving with respect to $\left(x_{B}^{A}, x_{B}^{B}, y_{B}\right)$ and that $B$ would accept.
} 
Proof. Given that there is no SSP equilibrium with delay if $\delta^{A}, \delta^{B}<1$, it is sufficient to show the upper hemicontinuity of the correspondence of allocations of SSP equilibria with immediate acceptance.

Consider then the correspondence $\Phi$ defined as

$$
\begin{array}{rr}
\Phi\left(x_{A}^{A}, x_{A}^{B}, y_{A}, x_{B}^{A}, x_{B}^{B}, y_{B} ; \delta^{A}, \delta^{B}\right)= \\
\arg \max _{0 \leq x^{A}, x^{B}, y} u^{A}\left(x^{A}, y\right) \times \arg \max _{0 \leq x^{A}, x^{B}, y} u^{B}\left(x^{B}, y\right) \\
D u^{B}\left(x^{B}, y\right)\left(\begin{array}{c}
x^{B}-e^{B} \\
y
\end{array}\right) \geq 0 & D u^{A}\left(x^{A}, y\right)\left(\begin{array}{c}
x^{A}-e^{A} \\
y
\end{array}\right) \geq 0 \\
u^{B}\left(x^{B}, y\right) \leq \delta^{B} u^{B}\left(x_{B}^{B}, y_{B}\right) & u^{A}\left(x^{A}, y\right) \geq \delta^{A} u^{A}\left(x_{A}^{A}, y_{A}\right) \\
x^{A}+x^{B}+M y=e^{A}+e^{B} & x^{A}+x^{B}+M y=e^{A}+e^{B} \\
\text { given }\left(x_{B}^{A}, x_{B}^{B}, y_{B}\right) & \text { given }\left(x_{A}^{A}, x_{A}^{B}, y_{A}\right)
\end{array}
$$

where $D u^{i}\left(x^{i}, y\right)$ stands for $\left(u_{x}^{i}\left(x^{i}, y\right), u_{y}^{i}\left(x^{i}, y\right)\right)$, for all $i=A, B$. Note that, by the Theorem of the Maximum,

$$
\begin{array}{r}
\arg \max _{0 \leq x^{A}, x^{B}, y} u^{A}\left(x^{A}, y\right) \\
D u^{B}\left(x^{B}, y\right)\left(\begin{array}{c}
x^{B}-e^{B} \\
y
\end{array}\right) \geq 0 \\
u^{B}\left(x^{B}, y\right) \geq \delta^{B} u^{B}\left(x_{B}^{B}, y_{B}\right) \\
x^{A}+x^{B}+M y=e^{A}+e^{B} \\
\text { given }\left(x_{B}^{A}, x_{B}^{B}, y_{B}\right)
\end{array}
$$

is a compact-valued, upper hemicontinuous correspondence that depends explicitly on $x_{B}^{B}, y_{B}$, and $\delta^{B}$ but also trivially on $x_{B}^{A}$ and $x_{A}^{A}, x_{A}^{B}, y_{A}, \delta^{A} .{ }^{10}$ And similarly for agent $B$ 's problem. Therefore, $\Phi$ is the cartesian product of compact-valued,

${ }^{10}$ Since $u^{A}$ depends continuously on $x^{A}, y$ and also trivially on $x_{A}^{A}, x_{A}^{B}, y_{A}, x_{B}^{A}, x_{B}^{B}, y_{B}, \delta^{A}, \delta^{B}$, and the correspondence defined by the constraints

$$
\begin{array}{r}
\Omega^{A}\left(x_{A}^{A}, x_{A}^{B}, y_{A}, x_{B}^{A}, x_{B}^{B}, y_{B}, \delta^{A}, \delta^{B}\right)=\left\{\left(x^{A}, x^{B}, y\right) \in \mathbb{R}^{2 n+m} \mid D u^{B}\left(x^{B}, y\right)\left(\begin{array}{c}
x^{B}-e^{B} \\
y
\end{array}\right) \geq 0\right. \\
u^{B}\left(x^{B}, y\right) \geq \delta^{B} u^{B}\left(x_{B}^{B}, y_{B}\right) \\
\left.x^{A}+x^{B}+M y=e^{A}+e^{B}\right\}
\end{array}
$$

is continuous and compact-valued. 
upper hemicontinuous correspondences, and hence it is compact-valued and upper hemicontinuous itself. ${ }^{11}$

Consider the correspondence $\Gamma$ of fixed points of $\Phi$ as a function of $\delta^{A}, \delta^{B}$, i.e.

$$
\begin{aligned}
\Gamma\left(\delta^{A}, \delta^{B}\right)=\{ & \left(x_{A}^{A}, x_{A}^{B}, y_{A}, x_{B}^{A}, x_{B}^{B}, y_{B}\right) \in \mathbb{R}^{2(2 n+m)} \mid \\
& \left.\left(x_{A}^{A}, x_{A}^{B}, y_{A}, x_{B}^{A}, x_{B}^{B}, y_{B}\right) \in \Phi\left(x_{A}^{A}, x_{A}^{B}, y_{A}, x_{B}^{A}, x_{B}^{B}, y_{B} ; \delta^{A}, \delta^{B}\right)\right\} .
\end{aligned}
$$

Since $\Phi$ is compact-valued and upper hemicontinuous, then the correspondence mapping the fixed points of $\Phi\left(\cdot, \cdot, \cdot, \cdot, \cdot, \cdot, \delta^{A}, \delta^{B}\right)$ to each pair $\left(\delta^{A}, \delta^{B}\right)$ is upper hemicontinuous. ${ }^{12}$

Finally, note that $\Gamma$ is the correspondence of SSP equilibrium allocations (without delay). Since this correspondence is upper hemicontinuous, in particular at $\left(\delta^{A}, \delta^{B}\right)=(1,1)$ and, according to Theorem $1, \Gamma(1,1)$ is the set of Lindahl allocations, then the claim follows.

Q.E.D.

\section{Final REMARKS}

In this paper we consider the collective decision problem faced by two agents who must agree on the output level and financing of public goods, and we show how bargaining over tax schedules can provide a foundation for Lindahl equilibrium allocations. We think that this result provides new insights into the long standing problem of finding strategic foundations for the Lindahl equilibrium. In particular, it addresses the criticism that in the presence of personalized Lindahl prices agents cannot be price-takers, by explicitly letting the agents offer prices.

We limit the analysis to the two-agent, complete information case. We think that this case captures the essentials of the actual bargaining taking place in legislatures over the funding of public goods. Examples of interesting and important instances

${ }^{11}$ See Lemma A1 in Dávila and Eeckhout (2008).

${ }^{12}$ See Lemma A3 in Dávila and Eeckhout (2008). 
of two-party bargaining are negotiations in legislatures between government and opposition, between territorial entities in federal countries, or in bilateral international agreements.

A natural question is how to extend our bargaining game to a situation with more agents, as in the models considered by Harrington (1989), Baron and Ferejohn (1989), Banks and Duggan (2000), et al. Consider an $n \geq 3$ person society in which the agents alternate in the role of proposer in a fixed order. A proposal consists as in this paper of a price vector and a quota, and each agent other than the proposer sequentially decides whether to accept or not the proposal and in case of acceptance chooses a maximum acceptable level of public goods. We conjecture that a characterization of allocations corresponding to SSP equilibria without delay analogous to that given by equations (6) and (7) can be obtained in this setting, so that Theorems 1 and 2 still hold. We leave this question open for future research.

\section{APPENDIX}

Lemma 1. If $(x, y)$ solves

$$
\begin{array}{r}
\max u(x, y) \\
p \cdot(x-e, y) \leq 0 \\
\|(x-e, y)\| \leq q,
\end{array}
$$

where $\|\cdot\|$ stands for the Euclidean norm, then

$$
D u(x, y)\left(\begin{array}{c}
x-e \\
y
\end{array}\right) \geq 0,
$$

and conversely, if $x$ satisfies (A.2), then there exist $p, q$ for which $x$ solves (A.1).

Proof. Assume $q>0$, otherwise (A.2) is trivially satisfied. Since $x$ solves (A.1), then there exist $\lambda, \mu \geq 0$ such that

$$
\begin{array}{r}
D u(x, y)=\lambda p+\mu(x-e, y) \\
\lambda p(x-e, y)=0 \\
\mu\left[(x-e)^{t}(x-e)+y^{t} y-q^{2}\right]=0 .
\end{array}
$$

Therefore,

$$
\begin{aligned}
D u(x, y)(x-e, y) & =\lambda p(x-e, y)+\mu(x-e, y)(x-e, y) \mid \\
& =\mu(x-e, y)(x-e, y) \geq 0 .
\end{aligned}
$$


Conversely, if $x, y$ satisfies $D u(x, y)(x-e, y)=0$, let

$$
\begin{aligned}
\lambda & =1 \\
\mu & =0 \\
p & =D u(x, y) \\
q^{2} & =(x-e, y)(x-e, y) .
\end{aligned}
$$

If $x, y$ satisfies $D u(x, y)(x-e, y)>0$, let

$$
\begin{aligned}
\lambda & >0 \\
\mu & =\frac{D u(x, y)(x-e, y)}{(x-e, y)(x-e, y)} \\
p & =\frac{1}{\lambda}\left[D u(x, y)-\frac{D u(x, y)(x-e, y)}{(x-e, y)(x-e, y)}(x-e, y)\right] \geq 0 \\
q^{2} & =(x-e, y)(x-e, y),
\end{aligned}
$$

where the weak inequality follows from the fact that if $a \in \mathbb{R}_{++}^{n}$ and $b \notin \mathbb{R}_{+}^{n}$ are such that $a b>0$, then ${ }^{13}$

$$
a-\frac{a \cdot b}{b \cdot b} b \geq 0 .
$$

Q.E.D.

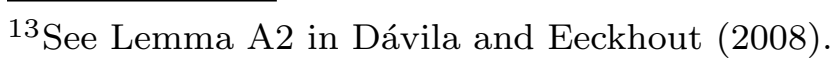




\section{REFERENCES}

Baron, D.P. and J.A. Ferejohn (1989), "Bargaining in Legislatures," American Political Science Review, 83, 1181-1206.

Banks, J. and J. Duggan (2000), "A Bargaining Model of Collective Choice," American Political Science Review, 94, 73-88.

Dávila, J. and J. Eeckhout (2008), "Competitive Bargaining Equilibrium," Journal of Economic Theory, 139, 269-294.

Groves, T. and J. Ledyard (1977), "Optimal Allocation of Public Goods: A Solution to the Free Rider' Problem," Econometrica, 45, 783-809.

Harrington, J. (1989), "The Advantageous Nature of Risk Aversion in a Threeplayer Bargaining Game where Acceptance of a Proposal Re- quires a Simple Majority," Economics Letters, 30, pp. 195-200.

Lindahl, E. (1919), "Just Taxation: A Positive Solution," reprinted in part in Classics in Theory of Public Finance, R. Musgrave and A. Peacock, editors (1958), McMillan, New York, pp. 168-176.

Rubinstein, A. (1982), "Perfect Equilibrium in a Bargaining Model," Econometrica, 50, $97-110$.

Thomson, W. (1999), "Economies with Public Goods: An Elementary Geometrical Exposition," Journal of Public Economic Theory, 1, 139-176.

Wicksell, K. (1896), "A New Principle of Just Taxation," reprinted in part in Classics in Theory of Public Finance, R. Musgrave and A. Peacock, editors (1958), McMillan, New York, pp. 72-118. 